YCTP-P16-92

May 1992

\title{
Contribution of Long Wavelength Gravitational Waves to the Cosmic Microwave Background Anisotropy
}

\author{
Martin White円 \\ Center for Theoretical Physics, Sloane Laboratory \\ Yale University, New Haven CT 06511
}

\begin{abstract}
We present an in depth discussion of the production of gravitational waves from an inflationary phase that could have occurred in the early universe, giving derivations for the resulting spectrum and energy density. We also consider the large-scale anisotropy in the cosmic microwave background radiation coming from these waves. Assuming that the observed quadrupole anisotropy comes mostly from gravitational waves (consistent with the predictions of a flat spectrum of scalar density perturbations and the measured dipole anisotropy) we describe in detail how to derive a value for the scale of inflation of $(1.5-5) \times 10^{16} \mathrm{GeV}$, which is at a particularly interesting scale for particle physics. This upper limit corresponds to a $95 \%$ confidence level upper limit on the scale of inflation assuming only that the quadrupole anisotropy from gravitational waves is not cancelled by another source. Direct detection of gravitational waves produced by inflation near this scale will have to wait for the next generation of detectors.
\end{abstract}

To appear in: Physical Review D

\footnotetext{
${ }^{1}$ Address after September 1, Center for Particle Astrophysics, University of California, Berkeley
} 


\section{Introduction}

It has long been realized that a period of inflation in the early universe would lead to production of a well defined spectrum of gravitational waves [1] (while inflation is not the only method of generating a stochastic background of horizon-sized waves [2], it is certainly the most well motivated). Since the waves decouple early they are a potentially good probe of conditions in the early universe. In fact as pointed out in [3, 4, 5, 7] such waves, which are fluctuations in the metric, can result in distortions of the cosmic microwave background radiation (CMBR) thus allowing information on the amplitude of the waves (and the parameters of the inflationary phase that produced them) to be derived from the measured CMBR anisotropy. This program proceeds in 3 steps. First the expected fluctuation spectrum is derived, then the consequences of such a spectrum for CMBR anisotropies are calculated. Finally comparing these predictions with the observations allows us to infer the parameters of an inflationary theory that produces such waves.

Recently an analysis of the implications of a CMBR anisotropy connected with gravitational waves from inflation was presented [8]. In this paper we fill in details of the results presented in [8] and further examine the issues addressed therein. A review of each of the 3 steps mentioned above will be given (many of the analytic results presented here have appeared in some form scattered in the literature, we have attempted to check, unify and reconcile previous estimates). The results can be compared to the recent COBE data [9] which gives the first positive measurement of anisotropies in the CMBR (previously this anisotropy had only been limited from above [10, 11]). On the theoretical side we give the details of derivations of some of the key results in the hope that these will be of use. We present in one place the derivation of the scale invariant spectrum predicted by exponential inflation (and a description of the scalar analogy), the energy density $\rho$ or equivalently $\Omega_{g}$, the CMBR anisotropy produced and the predicted temperature correlation function. We show how "redshift during horizon crossing" dramatically reduces the predicted $\Omega_{g}$ from their asymptotic value for waves re-entering the horizon during the matter dominated epoch. Our results for the multipole moments of the CMBR temperature fluctuation agree with I5] (though we use an approach due to [12] to derive the scale invariant spectrum) and are a factor of $\approx 2$ larger than [4]. With these results in hand we describe how the recent COBE observation is consistent with inflation and can limit the "scale near the end of inflation" assuming that the CMBR anisotropy is due, at least in part, to inflation-produced gravitational waves (the contribution from scalar density perturbations is constrained by the dipole anisotropy as we shall discuss). The scale turns out to be few $\times 10^{16} \mathrm{GeV}[8]$ - an interesting energy for particle physics.

The outline of the paper is as follows: section 2 establishes the notation and conventions. In sections 3 and 4 we discuss the scale-invariant spectrum predicted by exponential inflation and the spectral energy density. Section 5 is devoted to the derivation of the anisotropy generated by a stochastic spectrum of gravitational waves. The relation to the temperature correlation function measured by double- and triple-beam experiments (e.g. COBE) is derived in section 6 and the 
current measurement of the CMB anisotropy is compared to the theoretical predictions in section 7. Section 8 contains the conclusions.

\section{Cosmological history}

We will be interested in a cosmology which had an early period of inflation (where the dominant energy density resided in the vacuum). To be specific let us suppose that the universe underwent exponential inflation, then became radiation dominated and is currently in a matter dominated phase. The assumption of exponential inflation is a good approximation for "successful" inflation models [13] based on general relativity since the requirement of a "slow roll" period in the evolution of the inflaton field requires a very flat potential. The other possibility is that of power law inflation, where the scale factor grows like a power of time. This changes the energy density spectrum (putting more power in long wavelength modes) and enhances the CMBR anisotropies for small $l$ [5]. It seems difficult to have consistent power law inflation even in Brans-Dicke theory and we will not consider it further.

Our metric is of the usual $k=0$ Robertson-Walker form

$$
d s^{2}=-d t^{2}+R^{2}(t)\left(d x^{2}+d y^{2}+d z^{2}\right)=R^{2}(\tau)\left(-d \tau^{2}+d \vec{x}^{2}\right)
$$

where $d \tau=d t / R(t)$ is the conformal time. For an equation of state $p=q \rho$ the scale factor is a power of the conformal time, $R(\tau) \sim \tau^{2 /(1+3 q)}$. For later convenience we list below the scale factor, which we normalize to unity today, for the history described above along with the dominant form of energy density for each epoch. We assume that the transitions are sudden and match $R(\tau)$ and $\dot{R}(\tau)$ at the transition points (this will be a good enough approximation for our purposes) [12]. This matching implies that $\tau$ is discontinuous across each transition and $R(\tau)$ has the form

$$
R(\tau)=\left\{\begin{array}{cllc}
-(H \tau)^{-1} & \text { vacuum } & \tau \in & \left(-\infty,-\tau_{2}\right) \\
2 \tau_{1} \tau / \tau_{0}^{2} & \text { radiation } & \tau \in & \left(\tau_{2}, \tau_{1} / 2\right) \\
\tau^{2} / \tau_{0}^{2} & \text { matter } & \tau \in & \left(\tau_{1}, \tau_{0}\right)
\end{array}\right.
$$

where $H$ is the Hubble constant during inflation. Equating $\int_{0}^{\tau_{x}} d \tau$ with $\int_{0}^{t_{x}} d t / R(t)$ we find $\tau_{0}=3 t_{0}$ is the conformal time today and $\tau_{1}=\left(36 t_{1} t_{0}^{2}\right)^{1 / 3}$ is the conformal time at matter-radiation equality (both $\tau_{0}$ and $\tau_{1}$ refer to $\tau$ as measured in the matter dominated era - in this respect our notation differs from that in [12]). The conformal time at the end of inflation is $\tau_{2}=\tau_{0} / \sqrt{2 H \tau_{1}}$. The Hubble constant $H$ and vacuum energy density $V_{0}$ driving the inflation are related by

$$
H^{2}=\frac{8 \pi}{3} \frac{V_{0}}{m_{P l}^{2}}=\frac{8 \pi}{3} m_{P l}^{2} v
$$

where $v \equiv V_{0} / m_{P l}^{4}$. 
It is useful to have an expression for the size of a wave, relative to the horizon size. During a normal FRW expansion the coordinate radius of the horizon grows as $d r=d t / R(t)=d \tau$. If the period of inflation is long the coordinate radius of the horizon is very nearly zero at the beginning of the radiation dominated era. Thus at time $t_{*}$ the proper radius of the horizon is $\approx R\left(\tau_{*}\right) \tau_{*}$. If we consider a wave just entering the horizon at this time we find its comoving wavenumber, $k$, must satisfy

$$
\lambda_{\text {phys }}=R_{*} \tau_{*} \Rightarrow k \equiv \frac{2 \pi R}{\lambda_{\text {phys }}}=\frac{2 \pi}{\tau_{*}}
$$

This leads us to the following two limits. A wave with $k \tau \ll 2 \pi$ is well outside the horizon while a wave with $k \tau \gg 2 \pi$ is well within the horizon.

\section{The scalar analogy}

A classical gravitational wave in the linearized theory is a ripple on the background space-time

$$
g_{\mu \nu}=R^{2}(\tau)\left(\eta_{\mu \nu}+h_{\mu \nu}\right) \quad \text { where } \eta_{\mu \nu}=\operatorname{diag}(-1,1,1,1), \quad h_{\mu \nu} \ll 1
$$

In what follows we will work in transverse traceless (TT) gauge and denote the two independent polarization states of the wave as,$+ \times$. In the linear theory the TT metric fluctuations are gauge invariant (they can be related to components of the curvature tensor) [15]. We can write a plane wave with comoving wavenumber $|\vec{k}|$

$$
h_{\mu \nu}(\tau, \vec{x})=h_{\lambda}(\tau ; \vec{k}) e^{i \vec{k} \cdot \vec{x}} \epsilon_{\mu \nu}(\vec{k} ; \lambda)
$$

where $\epsilon_{\mu \nu}(\vec{k} ; \lambda)$ is the polarization tensor and $\lambda=+, \times$. The equation for the amplitude $h_{\lambda}(\tau ; \vec{k})$ is obtained by requiring the perturbed metric (5) satisfy Einstein's equations to $O(h)$. One finds [15]

$$
\ddot{h}_{\lambda}+2 \frac{\dot{R}}{R} \dot{h}_{\lambda}+k^{2} h_{\lambda}=0
$$

As noticed by Grishchuk [16] this is just the massless Klein-Gordon equation for a plane wave in the background space-time. Thus each polarization state of the wave behaves as a massless, minimally coupled, real scalar field, with a normalization factor of $\sqrt{16 \pi G}$ relating the two. We can use the following heuristic argument to fix the $\sqrt{16 \pi G}$ : in the linearized theory one can write the Hilbert action for the fluctuation using the contribution to the Ricci scalar from $h_{\mu \nu}$. From the derivation of the stress-energy tensor $T_{\mu \nu}^{(h)}$ [15] one sees $R^{(h)}=\frac{1}{4} h_{\mu \nu ; \rho} h^{\mu \nu ; \rho}$ so the corresponding Hilbert action is

$$
S_{H}^{(h)}=\frac{\sqrt{-g}}{16 \pi G} \frac{1}{2}\left[\left(\nabla h_{+}\right)^{2}+\left(\nabla h_{\times}\right)^{2}\right]
$$


which is the action for two real, massless, scalar fields $\phi_{+, \times}=(16 \pi G)^{-1 / 2} h_{+, \times}$as expected. Thus the study of quantum mechanical graviton production reduces to the study of the fluctuations of a scalar field in the curved background space-time, a factor $\sqrt{16 \pi G}$ relating the two cases.

\section{The spectrum and $\Omega_{g}$}

Quantum fluctuations have an important consequence in a cosmology with inflation. During inflation short wavelength quantum fluctuations will get red-shifted out of the horizon, after which they freeze in. In terms of (7) the freezing in of extrahorizontal modes is the statement that when wavelength is much larger than the horizon the $k^{2} h$ term is negligible and the solution is a constant amplitude: $\dot{h}=0$ (even before this limit is reached we note that the behaviour of waves outside the horizon is qualitatively different from the damped oscillatory behaviour of waves within it). When the mode re-enters the horizon at a much later epoch it appears as a long wavelength, classical gravitational wave (in analogy with the case of scalar fluctuations considered by [17]). One way of thinking of this [18 is that the number of quanta describing a state of constant amplitude grows as a power of the scale factor, the energy of each quantum being redshifted. Thus when the mode re-enters the horizon it represents a very large number of quanta.

The spectrum of gravitational waves generated by quantum fluctuations during the inflationary period can be derived by a sequence of Bogoliubov transformations relating creation and annihilation operators defined in the various phases: inflationary, radiation and matter dominated [12, 19]. The key idea is that for modes which have inflated outside the horizon the transitions between the phases are sudden and the universe will remain in the quantum state it occupied before the transition (treating each of the transitions as instantaneous is a good approximation for all but the highest frequency graviton modes). However the creation and annihilation operators that describe the particles in the state are related by a Bogoliubov transformation, so the quantum expectation value of any string of fields is changed (see e.g. [14 for a discussion of the calculation of Bogoliubov coefficients).

We calculate the statistical average of the ensemble of classical waves by considering the corresponding quantum average. The simplest examples to consider are the 2-point functions. For the quantum theory we calculate the scalar 2-point function, following [12]

$$
\left.\Delta \equiv \frac{k^{3}}{(2 \pi)^{3}} \int d^{3} x e^{i \vec{k} \cdot \vec{x}}\langle\psi|\phi(\vec{x}, \tau) \phi(\overrightarrow{0}, \tau)| \psi\rangle \quad \text { where }|\psi\rangle=\mid \text { de Sitter vac. }\right\rangle
$$

with the field

$$
\phi(\vec{x}, \tau)=\int d^{3} k\left(e^{i \vec{k} \cdot \vec{x}} a(k) \phi_{k}(\tau)+e^{-i \vec{k} \cdot \vec{x}} a^{\dagger}(k) \phi_{k}^{*}(\tau)\right)
$$

where $\phi_{k}(\tau)$ is a properly normalized solution of $(\overline{0})$. For the case of interest, a matter dominated 
universe today, $\phi_{k}$ is related to a Hankel function of order $3 / 2$

$$
\phi_{k}(\tau)=\frac{e^{-i k \tau}}{\sqrt{2 k}(2 \pi)^{3 / 2} R(\tau)}\left(1-\frac{i}{k \tau}\right)
$$

In the exponentially inflating phase $\phi_{k}$ has the same functional form (with different $R(\tau)$ ) and in the radiation dominated phase the form is again as (11), with appropriate $R(\tau)$ and also with the factor in parentheses absent.

The quantum 2-point function is obtained by using the Bogoliubov coefficients relating the creation and annihilation operators $a$ and $a^{\dagger}$ of the field in the 3 phases

$$
\begin{aligned}
a_{\text {rad }} & =c_{1}(\vec{k}) a_{i n f}(\vec{k})+c_{2}^{*}(\vec{k}) a_{i n f}^{\dagger}(-\vec{k}) \\
a_{m a t} & =c_{3}(\vec{k}) a_{i n f}(\vec{k})+c_{4}^{*}(\vec{k}) a_{i n f}^{\dagger}(-\vec{k})
\end{aligned}
$$

We are interested in waves which are still well outside the horizon at the time of matter-radiation equality $\left(k \tau_{1} \ll 2 \pi\right)$ since these will give the largest contribution to the CMBR anisotropy today. Matching the field and its first derivative at $\tau_{2}, \tau_{1}$ in the limit $k \tau \ll 2 \pi$ we find

$$
c_{1} \approx-c_{2} \approx \frac{-H \tau_{1}}{\left(k \tau_{0}\right)^{2}} \quad, \quad c_{3} \approx c_{4}^{*} \approx \frac{-3 i H}{2 k^{3} \tau_{0}^{2}}
$$

which is, correcting for differences in conventions, in agreement with [12]. Using (11, 13, 14) in (9) one obtains, for waves re-entering the horizon in the matter dominated era

$$
\Delta=\frac{H^{2}}{2(2 \pi)^{3}}\left[\frac{3 j_{1}(k \tau)}{k \tau}\right]^{2} \Rightarrow \Delta_{G W}=\frac{H^{2}}{\pi^{2} m_{P l}^{2}}\left[\frac{3 j_{1}(k \tau)}{k \tau}\right]^{2} \delta_{\lambda \lambda^{\prime}}
$$

where in the last step the scalar result was multiplied by $16 \pi G$ to obtain the corresponding result for gravitational waves. The corresponding expression for waves entering during the matter dominated era can be obtained by replacing the factor in brackets by $j_{0}(k \tau)$ as one would expect (since $j_{0}$ gives the right $\tau$ dependence from (7) and the amplitude as $k \tau \rightarrow 0$ should be the same).

We match this to our classical ensemble of gravitational waves, $h_{\lambda}(\tau ; \vec{k})$, by writing

$$
h_{\lambda}(\tau ; \vec{k})=A(k) a_{\lambda}(\vec{k})\left[\frac{3 j_{1}(k \tau)}{k \tau}\right] \quad \text { with } \quad \lambda=+, \times
$$

where the term in $[\cdots]$ is the real solution to (7) in the matter dominated phase and $a_{\lambda}(\vec{k})$ is a random variable with statistical expectation value

$$
\left\langle a_{\lambda}(\vec{k}) a_{\lambda^{\prime}}(\vec{q})\right\rangle=k^{-3} \delta^{(3)}(\vec{k}-\vec{q}) \delta_{\lambda \lambda^{\prime}}
$$


The 2-point function analogous to $\Delta$ in this case is simply

$$
\Delta_{\lambda \lambda^{\prime}}^{\prime}=A^{2}(k)\left[\frac{3 j_{1}(k \tau)}{k \tau}\right]^{2} \delta_{\lambda \lambda^{\prime}}
$$

Matching the quantum and classical 2-point functions gives us the well known prediction for the ( $k$-independent) spectrum of gravitational waves generated by inflation

$$
A^{2}(k)=\frac{H^{2}}{\pi^{2} m_{P l}^{2}}=\frac{8}{3 \pi} v
$$

The reason for the explicit factor of $k^{3}$ introduced in the definition of $\Delta$ (9) can be seen by considering the expression for the spectral energy density in (classical) gravitational waves. In our notation

$$
\rho=\frac{k_{\text {phys }}^{2}}{16 \pi G}\left\langle h_{+}^{2}+h_{\times}^{2}\right\rangle
$$

where $\langle\cdots\rangle$ indicates an average over many wavelengths/periods as well as an average over the stochastic variable $a_{\lambda}(\vec{k}) . k_{\text {phys }}=k / R(\tau)$ is the physical wavenumber. For a fixed (non-stochastic) spectrum of waves we could write the average over space as an integral over Fourier modes using Parseval's theorem and then relate the "power spectrum" $\left|h_{+, \times}(k)\right|^{2}$ to the correlation function (9). With (16) and (17) as defined the stochastic average produces the same result. Explicitly

$$
k \frac{d \rho_{\lambda}}{d k}=\frac{k_{p h y s}^{2}}{4 G} \overline{\Delta_{\lambda \lambda}^{\prime}} \quad \lambda=+, \times
$$

which goes as $R^{-4}(\tau)$ as expected (an overbar denotes averaging). Up to the time evolution factor $\overline{\left(3 j_{1}(k \tau) / k \tau\right)^{2}}$ for matter and $\overline{j_{0}(k \tau)}$ for radiation dominated phases this agrees with [20]. Including this factor for waves re-entering during the matter dominated phase (i.e. considering evolution as the wave enters the horizon) leads to a significant suppression of $\rho$. Writing the energy density as a fraction of the closure density one finds, for waves just entering the horizon [8],

$$
\left.\Omega_{g} \equiv \sum_{\lambda=+, \times} \frac{k}{\rho_{c}} \frac{d \rho_{\lambda}}{d k}\right|_{k=2 \pi / \tau}=\left\{\begin{array}{cc}
16 v / 9 & \mathrm{RD} \\
v / \pi^{2} & \mathrm{MD}
\end{array}\right.
$$

In deriving this use has been made of the fact that $k_{p h y s}=1(2) \pi H_{H C}$ for waves crossing the horizon during the matter (radiation) dominated phase $\left(H_{H C}\right.$ is the Hubble constant at the time the wave re-enters the horizon).

Will we be able to see this energy density with terrestrial or astrophysical gravitational wave detector? The most sensitive gravitational wave limit currently comes from the timing of millisecond 
pulsars. This is sensitive to "short" wavelengths (periods of order years) and puts an upper limit on the energy density in such waves $\Omega_{g}<9 \times 10^{-8}(68 \%)$ (this limit improves as the fourth power of the observing time: $T_{o b s}^{4}$ ] [28]. Such short wavelength modes entered the horizon during the radiation dominated era. If we write the Hubble constant today $H_{0}=100 \mathrm{~h} \mathrm{~km} / \mathrm{s} / \mathrm{Mpc}$ then these waves contribute to $\Omega_{g}$ today an amount suppressed by a factor of $\rho_{\text {rad }} / \rho_{c} \approx 4 \times 10^{-5} h^{-2}$ compared to horizon crossing since they redshift with one extra power of $R$ compared to matter. For values of $v \sim 10^{-9}$, consistent with the anisotropy of the CMBR (see later), the energy density per logarithmic frequency interval in short wavelength modes is predicted to be $\Omega_{g} \sim 10^{-13}$ [8], which is $\sim 6$ orders of magnitude lower than the millisecond pulsar limit. Even the proposed LIGO detector [29] which has a sensitivity of $\Omega_{g} \sim 10^{-11}$ is short of the mark by 2 orders of magnitude. Thus we expect that a positive detection of the signal will require a significant advance in technology.

Since the predicted CMBR anisotropy multipoles, up to $l \sim 9$, from gravitational waves and scale invariant scalar fluctuations are very similar [7] and direct detection of gravitational waves is still some way off the outlook for determining unambiguously that a significant fraction of the observed CMBR anisotropy comes from gravitational waves seems bleak.

\section{Anisotropy from the stochastic background}

It is conventional to expand the CMBR temperature anisotropy in spherical harmonics

$$
\frac{\delta T}{T}(\theta, \phi)=\sum_{l m} a_{l m} Y_{l m}(\theta, \phi)
$$

We can calculate the prediction of a given spectrum of gravitational waves in terms of the $a_{l m}$. The temperature fluctuation due to a gravitational wave $h_{\mu \nu}$ can be found in the linearized theory to be 23

$$
\frac{\delta T}{T}=-\frac{1}{2} \int_{e}^{r} d \Lambda \frac{\partial h_{\mu \nu}(\tau, \vec{x})}{\partial \tau} \hat{x}^{\mu} \hat{x}^{\nu}
$$

where $\Lambda$ is a parameter along the unperturbed path and the lower (upper) limit of integration represents the point of emission (reception) of the photon.

Now we project out a multipole and calculate the rotationally symmetric quantity

$$
\left\langle a_{l}^{2}\right\rangle \equiv\left\langle\sum_{m}\left|a_{l m}\right|^{2}\right\rangle=\sum_{m} \int d \Omega d \Omega^{\prime} Y_{l m}^{*}(\Omega) Y_{l m}\left(\Omega^{\prime}\right)\left\langle\frac{\delta T}{T}(\Omega) \frac{\delta T}{T}\left(\Omega^{\prime}\right)\right\rangle
$$

where using $(6,17,24)$ we have for a spectrum of waves

$$
\begin{aligned}
& \left\langle\frac{\delta T}{T}(\Omega) \frac{\delta T}{T}\left(\Omega^{\prime}\right)\right\rangle=\frac{1}{4} \sum_{\lambda=+, \times} \int \frac{d^{3} k}{k^{3}}\left[\int d \Lambda \dot{h}(\tau ; k) e^{i \vec{k} \cdot \vec{x}} \epsilon_{\mu \nu}(\vec{k} ; \lambda) \hat{x}^{\mu} \hat{x}^{\nu}\right] \times \\
& {\left[\int d \Lambda^{\prime} \dot{h}\left(\tau^{\prime} ; k\right) e^{i \vec{k} \cdot \overrightarrow{x^{\prime}}} \epsilon_{\mu \nu}(\vec{k} ; \lambda){\hat{x^{\prime}}}^{\mu}{\hat{x^{\prime}}}^{\nu}\right]^{*}}
\end{aligned}
$$


and an overdot represents a derivative with respect to $\tau$. We use the rotational symmetry of $a_{l}^{2}$ to make the replacement

$$
\sum_{m} Y_{l m}^{*}(\Omega) Y_{l m}\left(\Omega^{\prime}\right)=\sum_{m} Y_{l m}^{*}\left(\Omega_{k x}\right) Y_{l m}\left(\Omega_{k x}^{\prime}\right)
$$

where $\Omega_{k x}$ indicates that the angles are defined with respect to $\hat{k}$. We can evaluate directly

$$
\epsilon_{\mu \nu}(\vec{k} ; \lambda) \hat{x}^{\mu} \hat{x}^{\nu}=\left(\delta_{\lambda}^{+} \cos (2 \phi)+\delta_{\lambda}^{\times} \sin (2 \phi)\right) \sin ^{2} \theta
$$

where $\theta, \phi$ are the usual spherical angles relating $\hat{k}$ and $\hat{x}$.

First concentrate on the angular integrations. Expanding the exponential

$$
e^{i \vec{k} \cdot \vec{x}}=\sum_{n=0}^{\infty} i^{n}(2 n+1) j_{n}(k x) P_{n}\left(\cos \theta_{k x}\right)
$$

it is not hard to show (formula 7.125 of Gradshteyn and Ryzhik [24] is useful) that

$$
\begin{aligned}
I_{l m}(k, x) & \equiv \int d \Omega_{k x} Y_{l m}^{*}\left(\Omega_{k x}\right) e^{i \vec{k} \cdot \vec{x}} \epsilon_{\mu \nu}(\vec{k} ; \lambda) \hat{x}^{\mu} \hat{x}^{\nu} \\
& =\pi \sqrt{\frac{2 l+1}{4 \pi} \frac{(l+2) !}{(l-2) !}} \mathcal{H} \sum_{n} i^{n}(2 n+1) j_{n}(k x)\left(c_{-2} \delta_{l-2}^{n}+c_{0} \delta_{l}^{n}+c_{2} \delta_{l+2}^{n}\right)
\end{aligned}
$$

where

$$
\begin{aligned}
\mathcal{H} & =\left(\delta_{\lambda}^{+}-i \delta_{\lambda}^{\times}\right) \delta_{m}^{+2}+\left(\delta_{\lambda}^{+}+i \delta_{\lambda}^{\times}\right) \delta_{m}^{-2} \\
1 / c_{-2} & =(2 l-1)(2 l+1)(l-3 / 2) \\
1 / c_{0} & =-\frac{1}{2}(2 l-1)(2 l+3)(l+1 / 2) \\
1 / c_{+2} & =(2 l+1)(2 l+3)(l+5 / 2)
\end{aligned}
$$

Inserting this back into the expression for $a_{l}^{2}$ and using (16)

$$
\left\langle a_{l}^{2}\right\rangle=\frac{1}{4} \sum_{m} \sum_{\lambda=+, \times} \int \frac{d k d \Omega_{k}}{k}\left|\int d \Lambda A(k) \frac{d}{d \tau}\left(\frac{3 j_{1}(k \tau)}{k \tau}\right) I_{l m}(k, x)\right|^{2}
$$

The integral over the path $d \Lambda$ can be parameterized by the distance from the origin along the line of sight, $r$, so $|\vec{x}(r)|=r$ and $\tau(r)=\tau_{0}-r$. We will defer consideration of the limits of the $k$ integral for the moment. Thus

$$
\left\langle a_{l}^{2}\right\rangle=36 \pi^{2}(2 l+1) \frac{(l+2) !}{(l-2) !} \int k d k A^{2}(k)\left|F_{l}(k)\right|^{2}
$$


where the function $F_{l}(k)$ is defined as

$$
F_{l}(k) \equiv \int_{0}^{\tau_{0}-\tau_{1}} d r\left(\frac{d}{d(k \tau)} \frac{j_{1}(k \tau)}{k \tau}\right)\left[\frac{j_{l-2}(k r)}{(2 l-1)(2 l+1)}+\frac{2 j_{l}(k r)}{(2 l-1)(2 l+3)}+\frac{j_{l+2}(k r)}{(2 l+1)(2 l+3)}\right]
$$

Accounting for the factor of two difference between the definitions of $A^{2}(k)$ this is precisely the result of [5], and is a factor of $\approx 2$ larger than the earlier result of [4]. The integral in equation (37) can be expressed in terms of elementary functions and the sine integral but the result is very cumbersome and is not presented here.

For waves entering the horizon during the matter dominated regime the upper limit of the $k$ integration is $2 \pi / \tau_{1}$. The results are very insensitive to the exact choice of upper limit since the integral is dominated by small $k \approx 2 \pi / \tau_{0}$, i.e. waves that have recently entered the horizon. For very high frequency modes the transition between phases of the universe is no longer sudden and graviton production is suppressed. Taking this high frequency cutoff to be $k=2 \pi / \tau_{1}$ (i.e. restricting attention to waves that entered the horizon during the matter dominated era) introduces negligible error. The lower limit of the $k$ integral is zero, since waves of arbitrarily long wavelength can contributef. From the form of the integrand however one can see that the contribution to $\left\langle a_{l}^{2}\right\rangle$ tends to zero as $k^{n}(n \geq 1)$ as $k \tau \rightarrow 0$. Thus the contribution from very long wavelength modes is suppressed as one might expect on physical grounds.

One can now calculate the predicted $\left\langle a_{l}^{2}\right\rangle$ for any spectrum $A(k)$ of gravitational waves from (36). For the scale-invariant spectrum (19) of exponential inflation the $\left\langle a_{l}^{2}\right\rangle$ are shown in table 11. The calculation of the expectation value $\left\langle a_{l}^{2}\right\rangle$ is not the end of the story however. Before we compare predictions with observations we must also consider the statistical properties of $a_{l}^{2}$. The fact that the "weakly coupled" nature of inflation predicts the $a_{l m}$ to be independent makes this problem tractable. Given that each of the $a_{l m}$ are independent Gaussian random variables the probability distribution for each $a_{l}^{2}$, whose mean $\left\langle a_{l}^{2}\right\rangle$ we calculated, is of the $\chi^{2}$ form and can be written

$$
P(y) d y=\frac{y^{l-1 / 2} e^{-y}}{\Gamma\left(l+\frac{1}{2}\right)} d y \quad \text { where } \quad y=\frac{2 l+1}{2} \frac{a_{l}^{2}}{\left\langle a_{l}^{2}\right\rangle}
$$

This agrees with [6]. One can calculate the confidence levels for $a_{l}^{2}$ in terms of the incomplete gamma function (values for the 68, 90 and 95\% (lower) confidence levels can be found in table 1). We note in passing that the modal value of $a_{l}^{2}$ for any universe is $(2 l-1) /(2 l+1)$ times the mean.

\section{The correlation function}

Limits quoted on the multipole moments $a_{l}^{2}$ can be directly compared to (36) to constrain the inflationary theory. However many experiments (and importantly COBE) are of the double- or

\footnotetext{
${ }^{2}$ We thank Mark Wise and Vince Moncrief for very helpful discussions on this point.
} 
triple-beam type and do not simply measure any one multipole moment but can constrain the correlation function [9, 20]

$$
C\left(\theta_{21} ; \sigma\right) \equiv\left\langle\frac{\delta T}{T}\left(\hat{x}_{1} ; \sigma\right) \frac{\delta T}{T}\left(\hat{x}_{2} ; \sigma\right)\right\rangle_{21}
$$

where the average is over all positions $\hat{x}_{1}, \hat{x}_{2}$ on the sky with $\hat{x}_{1} \cdot \hat{x}_{2}$ fixed, and $\sigma$ is a measure of the angular response of the detector. We shall take the angular response to be Gaussian [21]

$$
d R(\theta, \phi)=\frac{\theta d \theta d \phi}{2 \pi \sigma^{2}} \exp \left[-\frac{\theta^{2}}{2 \sigma^{2}}\right]
$$

where $\theta, \phi$ are the angles relative to the beam direction and $\sigma \ll 1$ which justifies the use of the small angle expansion. We define the "smeared" $\delta T / T$ by

$$
\begin{aligned}
\frac{\delta T}{T}\left(\hat{x}_{1} ; \sigma\right) & \equiv \sum_{l m} a_{l m} \int d \Omega^{\prime} R\left(\Omega^{\prime}\right) Y_{l m}(\Omega) \\
& =\sum_{l m} a_{l m}\left(\int_{0}^{\infty} d x P_{l}\left(1-\sigma^{2} x\right) e^{-x}\right) Y_{l m}\left(\hat{x}_{1}\right)
\end{aligned}
$$

where $\Omega^{\prime}$ is the direction $\Omega$ measured relative to $\hat{x}_{1}$ and we have approximated $\cos \theta^{\prime} \approx 1-\theta^{\prime 2} / 2$ for small $\theta^{\prime}$. Note that the term in parenthesis suppresses the higher $l$ modes as we would expect physically. We can expand this term for small $\sigma^{2}$

$$
\begin{aligned}
\int_{0}^{\infty} d x P_{l}\left(1-\sigma^{2} x\right) e^{-x} & =\sum_{n=0}^{\infty}\left(-\sigma^{2}\right)^{n} P_{l}^{(n)}(1) \\
& =\sum_{n=0}^{l}\left(-\sigma^{2}\right)^{n} \frac{1}{2^{n} n !} \frac{(l+n) !}{(l-n) !}
\end{aligned}
$$

Making the approximation $(l+n) ! /(l-n) ! \approx(l+1 / 2)^{2 n}$ for large $l$ we can write the suppression term as: $\exp \left[-(l+1 / 2)^{2} \sigma^{2} / 2\right]$ (and at the same level of approximation we can replace $(l+1 / 2)^{2}$ with $l(l+1)$ as used by [9]). This exponential suppression proves a good approximation for $\sigma<0.1$ which is the range for most detectors. A simple exercise in manipulating spherical tensors allows us to write

$$
\left\langle Y_{l m}\left(\hat{x}_{1}\right) Y_{l^{\prime} m^{\prime}}^{*}\left(\hat{x}_{2}\right)\right\rangle_{21}=\frac{1}{4 \pi} \delta_{l l^{\prime}} \delta_{m m^{\prime}} P_{l}\left(\hat{x}_{1} \cdot \hat{x}_{2}\right)
$$

so we can express the correlation function (39) in a simple from as (see [9, 20])

$$
C\left(\hat{x}_{1} \cdot \hat{x}_{2} ; \sigma\right)=\frac{1}{4 \pi} \sum_{l=0}^{\infty} a_{l}^{2} P_{l}\left(\hat{x}_{1} \cdot \hat{x}_{2}\right) e^{-(l+1 / 2)^{2} \sigma^{2}}
$$


The predicted correlation function using the moments of table 1 is shown in figure 1 for a gaussian beam width of $\sigma=10^{\circ}$. The error bars represent the upper and lower limit of the $68 \%$ confidence region arrived at using (38) for the distribution of $a_{l}^{2} / v$ (there is no simple analytic form for the probability distribution of $C(\theta, \sigma)$ so figure 11 was obtained from a Monte-Carlo) and the solid line is $C(\theta, \sigma)$ evaluated using $a_{l}^{2}=\left\langle a_{l}^{2}\right\rangle$. The correlation function inferred from the COBE sky maps [9] is in agreement with the form shown in figure 1.

\section{Comparison with observations}

The recent results of COBE can be summarized as a non-zero value of the quadrupole moment $a_{2}^{2}$. If we require a Harrison-Zel'dovich spectrum the quadrupole, from the $Q_{r m s-P S}$ value, is measured to be [8, 9]

$$
a_{2}^{2}=(4.7 \pm 2) \times 10^{-10}
$$

An early inflationary phase produces not only gravitational waves but also a scale invariant spectrum of scalar density perturbations. To limit the contribution of these latter to $a_{2}^{2}$ we can require the induced dipole from long wavelength scalar modes not greatly exceed the observed dipole anisotropy. For a given (e.g. flat) spectrum one can use this to place limits on all the higher multipoles. At the $90 \%$ confidence level an upper limit of $a_{2}^{2} \approx 2 \times 10^{-10}$ [7] has been derived (ignoring the transfer function corrections). Equivalently fitting the observed clustering to a primordial spectrum yields $a_{2}^{2} \approx(2-10) \times 10^{-11}$ 225. Such estimates suggest a large fraction of the quadrupole anisotropy may be due to long wavelength gravitational waves.

Let us assume that the observed quadrupole is due entirely to gravitational waves. Using $\left\langle a_{2}^{2}\right\rangle / v$ from table 1 and including the distribution for the measured $a_{2}^{2}$ (gaussian) and predicted $a_{2}^{2} / v(38)$ one can infer a distribution for $v$. The result obtained from a Monte-Carlo is shown in figure 2. This corresponds to a mean $v=6.1 \times 10^{-11}$, and a modal $v=4 \times 10^{-11}$ with non-gaussian errors. The allowed range of $v$ from figure 2 can be summarized as [8]

$$
\begin{aligned}
& 2.3 \times 10^{-11}<v<1.5 \times 10^{-10} 68 \% C L \\
& 2.5 \times 10^{-12}<v<3.7 \times 10^{-10} 95 \% C L
\end{aligned}
$$

One can scale these results directly to obtain values for $v$ if gravitational wave contribution to $a_{2}^{2}$ is not $100 \%$, the rest being made up or shielded by scalar fluctuations for example.

Converting to a more familiar energy scale $M=\left(v^{1 / 4} M_{P l}\right)$ the $95 \%$ confidence level range above is $M \in(1.5-5.2) \times 10^{16} \mathrm{GeV}$, with a best fit $M=3 \times 10^{16} \mathrm{GeV}$. The upper limit quoted corresponds to a strict upper limit on the scale of inflation assuming that the quadrupole moment due to gravitational waves is not being cancelled to any significant degree by other sources (e.g. scalar density perturbations). A large cancellation between two sources of anisotropy would be unlikely. 
No realistic model of inflation currently exists, however most are based on the idea that a phase transition occurred in the early universe to produce the vacuum energy density to drive the inflation. If such a transition were to occur then the natural scale would seem to be the scale of unification of the coupling constants in the context of a Grand Unified Theory (GUT) (an exception is the case of chaotic inflation). The recent precision measurements from LEP have allowed us to sharpen our predictions for this scale. Assuming a supersymmetric GUT with the supersymmetry breaking scale $\approx 1 \mathrm{TeV}$, one predicts [26] a unification scale of $(1-3.6) \times 10^{16} \mathrm{GeV}$. For a theory based on $\mathrm{SO}(10)$ one finds $M=10^{15.8 \pm .22} \mathrm{GeV}$ [27]. The limits from proton decay experiments are very theory dependent, however they give a lower bound on $M$. The current limits are near or larger than $10^{15} \mathrm{GeV}$ for fashionable GUT theories.

Unless there is significant fine tuning or hierarchies in the theory the vacuum energy density associated with a transition from a GUT with scale $M_{G}$ is $V_{0}=\left(\kappa M_{G}\right)^{4}$ where $\kappa$ is a (calculable) number usually in the range $\kappa^{4}=.01-1$. This puts the GUT scale very close to the scale of inflation inferred above - a "numerical coincidence" which is both suggestive and exciting.

\section{Conclusions}

As the observations of CMBR anisotropies are refined and other gravitational wave detectors come on line the results presented here can allow a comparison of theoretical predictions and experimental results, which can help to constrain models of inflation and early universe physics.

The recent COBE results allow the possibility that the observed quadrupole anisotropy results from gravitational waves generated during inflation with a scale of $(1.5-5.2) \times 10^{16} \mathrm{GeV}$ - a very interesting range of energies. If the anisotropy is due to gravitational waves, confirmation will

probably not come from existing or currently planned gravitational wave detectors. Further results are eagerly awaited.

I would like to thank L. Krauss, V. Moncrief, M. Turner and M. Wise for extremely useful conversations. I also wish to thank E. Gates for comments on the manuscript. 


\section{References}

[1] A.A. Starobinsky, JETP Lett. 30 (1979) 682

[2] L.M. Krauss, Gravitational Waves from Global Phase Transitions, Phys. Lett. B, in press.

[3] V.A. Rubakov, M.V. Sazhin and A.V. Veryaskin, Phys. Lett. B115 (1982) 189; A.A. Starobinsky, Sov. Astron. Lett. 9 (1983) 302; A.A. Starobinsky, Sov. Astron. Lett. 11 (1985) 133

[4] R. Fabbri and M.D. Pollock, Phys. Lett. B125 (1983) 445; R. Fabbri in Proceedings of the International School of Physics, Enrico Fermi, 1982, course 86, ed. F. Melchiorri and R. Ruffini (North-Holland)

[5] L.F. Abbott and M.B. Wise, Nucl. Phys. B244 (1984) 541

[6] L.F. Abbott and M.B. Wise, Ap. J. 282 (1984) L47

[7] L.F. Abbott and R. Schaefer, Ap.J. 308 (1986) 546

[8] L.M. Krauss and M. White, Yale preprint, YCTP-P15-92, submitted to Phys. Rev. Lett.

[9] G.F. Smoot et al. , COBE preprint 92-04, submitted to Ap.J.; C.L. Bennet et al., COBE preprint 92-05, submitted to Ap.J.

[10] G.F. Smoot et al., Ap. J. 371 (1991) L1

[11] D.J. Fixsen, E.S. Chang and D. Wilkinson, Phys. Rev. Lett. 50 (1983) 620; P.M. Lubin et. al., Ap. J. 298 (1985) L1

[12] L.F. Abbott and D.D. Harari, Nucl. Phys. B264 (1986) 487

[13] P. Steinhardt and M.S. Turner, Phys. Rev. D29 (1984) 2162

[14] R.H. Brandenberger, Rev. Mod. Phys. 57 (1985) 1

[15] C.W. Misner, K.S. Thorne and J.A. Wheeler, "Gravitation", (W.H. Freeman, 1973); S. Weinberg, "Gravitation and Cosmology", (Wiley ,1972)

[16] L.P. Grishchuk, Sov.Phys. JETP 40 (1975) 409; L.P. Grishchuk, Ann. N.Y. Acad. Sci. 302 (1977) 439

[17] A. Guth and S.-Y. Pi, Phys. Rev. D32 (1985) 1899 
[18] L.M. Krauss, private communication

[19] V. Sahni, Phys. Rev. D42 (1990) 453

[20] E.W. Kolb and M.S. Turner, "The Early Universe", Frontiers in Physics vol 69, Addison-Wesley (1990).

[21] M. Wilson and J. Silk, Ap. J. 243 (1981) 14

[22] B. Allen, Phys. Rev. D37 (1988) 2078

[23] R.K. Sachs and A.M. Wolfe, Ap.J. 147 (1967) 73

[24] I.S. Gradshteyn and I.M. Ryzhik, "Table of Integrals, Series and Products", 4th ed., (Academic Press, 1980)

[25] J.R. Bond and G. Efstathiou, Mon. Not. R. Astr. Soc. 226 (1987) 655

[26] see S.Dimopoulos, S.A. Raby, F. Wilczek, Phys. Today, Oct. 1991, for early references; U. Amaldi, et al., Phys. Lett. B 260 (1991) 447; P. Langacker, M. Luo, U. Penn. preprint (1991)

[27] R.N. Mohapatra and M.K. Parida, preprint UMD-PP-92-179

[28] B. Bertotti, B.J. Carr, M.J. Rees, Mon. Not. R. Ast. Soc. 203 (1983) 945; L.M. Krauss, Nature 313 (1985) 32; D. R. Stinebring et al, Phys. Rev. Lett. 65 (1990) 285

[29] see K.S. Thorne, in "300 Years of Gravitation", ed. by S.W. Hawking and W. Israel (Cambridge Univ. Press, 1989) 


\section{Figure Captions}

Figure 1: Results of a Monte-Carlo for the predicted correlation function $C(\theta ; \sigma)$ generated by a flat spectrum of gravitational waves. The error bars represent the upper and lower limits of the $68 \%$ confidence region arrived at using the predicted distribution of $a_{l}^{2} / v$. The solid curve is the correlation function calculated using $\left\langle a_{l}^{2}\right\rangle$. The beam width is taken to be $\sigma=10^{\circ}$.

Figure 2: The probability distribution for the scale $v \equiv V_{0} / m_{P l}^{4}$ of inflation as determined by MonteCarlo from the COBE measurement of the quadrupole anistropy assuming that the whole anistropy is due to gravitational waves.

\section{Tables}




\begin{tabular}{|c|c|c|}
\hline & & $a_{l}^{2} /\left\langle a_{l}^{2}\right\rangle$ \\
& & \\
$l$ & $\left\langle a_{l}^{2}\right\rangle / v$ & $68 / 90 / 95 \%$ CL \\
\hline 2 & 7.74 & $.63 / .32 / .23$ \\
3 & 4.25 & $.69 / .40 / .31$ \\
4 & 3.10 & $.73 / .46 / .37$ \\
5 & 2.50 & $.76 / .51 / .42$ \\
6 & 2.12 & $.78 / .54 / .45$ \\
7 & 1.85 & $.80 / .57 / .48$ \\
8 & 1.64 & $.81 / .59 / .51$ \\
9 & 1.48 & $.82 / .61 / .53$ \\
10 & 1.35 & $.83 / .63 / .55$ \\
\hline
\end{tabular}

Table 1: Multipole coefficients $a_{l}^{2}$ for modes $l=2-10$ predicted for a stochastic background of gravitational waves generated by exponential inflation. The 68, 90 and $95 \%$ (lower) confidence levels are also shown as fractions of $\left\langle a_{l}^{2}\right\rangle$. 